\title{
Memory: A Contribution to Experimental Psychology
}

\author{
Hermann Ebbinghaus (1885) \\ Translated by Henry A. Ruger \& Clara E. Bussenius (1913) \\ (Reprinted with permission)
}

doi : $10.5214 /$ ans. 0972.7531 .200408

\section{Our Knowledge Concerning Memory}

The language of life as well as of science in attributing a memory to the mind attempts to point out the facts and their interpretation somewhat as follows:

Mental states of every kind, - sensations, feelings, ideas, which were at one time present in consciousness and then have disappeared from it, have not with their disappearance absolutely ceased to exist. Although the inwardly-turned look may no longer be able to find them, nevertheless they have not been utterly destroyed and annulled, but in a certain manner they continue to exist, stored up, so to speak, in the memory. We cannot, of course, directly observe their present existence, but it is revealed by the effects which come to our knowledge with a certainty like that with which we infer the existence of the stars below the horizon. These effects are of different kinds.

In a first group of cases we can call back into consciousness by an exertion of the will directed to this purpose the seemingly lost states (or, indeed, in case these consisted in immediate sense-perceptions, we can recall their true memory images) that is, we can reproduce them voluntarily. During attempts of this sort, - that is, attempts to recollect - all sorts of images toward which our aim was not directed, accompany the desired images to the light of consciousness. Often, indeed, the latter entirely miss the goal, but as a general thing among the representations is found the one which we sought, and it is immediately recognised as something formerly experienced. It would be absurd to suppose that our will has created it anew and, as it were, out of nothing; it must have been present somehow or somewhere. The will, so to speak, has only discovered it and brought it to us again.

In a second group of cases this survival is even more striking. Often, even after years, mental states once present in consciousness return to it with apparent spontaneity and without any act of the will; that is, they are reproduced involuntarily. Here, also, in the majority of cases we at once recognise the returned mental state as one that has already been experienced; that is, we remember it. Under certain conditions, however, this accompanying consciousness is lacking, and we know only indirectly that the "now" must be identical with the "then"; yet we receive in this way a no less valid proof for its existence during the intervening time. As more exact observation teaches us, the occurrence of these involuntary reproductions is not an entirely random and accidental one. On the contrary they are brought about through the instrumentality of other immediately present mental images. Moreover they occur in certain regular ways which in general terms are described under the so-called 'laws of association.'

Finally there is a third and large group to be reckoned with here. The vanished mental states give indubitable proof of their continuing existence even if they themselves do not return to consciousness at all, or at least not exactly at the given time. Employment of a certain range of thought facilitates under certain conditions the employment of a similar range of thought, even if the former does not come before the mind directly either in its methods or in its results. The boundless domain of the effect of accumulated experience belongs here. This effect results from the frequent conscious occurrence of any condition or process, and consists in facilitating the occurrence and progress of similar processes. This effect is not fettered by the condition that the factors constituting the experience shall return in toto to consciousness. This may incidentally be the case with a part of them; it must not happen to a too great extent and with too great clearness, otherwise the course of the present process will immediately be disturbed. Most of these experiences remain concealed from consciousness and yet produce an effect which is significant and which authenticates their previous existence.

\section{Section 2. Memory in its Dependence}

Along with this bare knowledge of the existence of memory and its effects, there is abundant knowledge concerning the conditions upon which depend the vitality of that inner survival as well as the fidelity and promptness of the reproduction.

How differently do different individuals behave in this respect! One retains and reproduces well; another, poorly. And not only does this comparison hold good when different individuals are compared with each other, but also when different phases of the existence of the same individual are compared: morning and evening, youth and old age, find him different in this respect.

Differences in the content of the thing to be reproduced are of great influence. Melodies may become a source of torment by the undesired persistency of their return. Forms and colors are not so importunate; and if they do return, it is with noticeable loss of clearness and certainty. The musician writes for the orchestra what his inner voice sings to him; the painter rarely relies without disadvantage solely upon the images which his inner eye presents to him; nature gives him his forms, study governs his combinations of them. It is with something of a struggle that past states of feeling are realized; when realized, and this is often only through the instrumentality of the movements which accompanied them, they are but pale shadows of themselves. Emotionally true singing is rarer than technically correct singing.

If the two foregoing points of view are taken together - differences in individuals and differences in content - an endless number of differences come to light. One individual overflows with poetical reminiscences, another directs symphonies from memory, while numbers and formulae, which come to a third without effort, slip away from the other two as from a polished stone.

Very great is the dependence of retention and reproduction upon the intensity of the attention and interest which were attached to the mental states the first time they were present. 
The burnt child shuns the fire, and the dog which has been beaten runs from the whip, after a single vivid experience. People in whom we are interested we may see daily and yet not be able to recall the color of their hair or of their eyes.

Under ordinary circumstances, indeed, frequent repetitions are indispensable in order to make possible the reproduction of a given content. Vocabularies, discourses, and poems of any length cannot be learned by a single repetition even with the greatest concentration of attention on the part of an individual of very great ability. By a sufficient number of repetitions their final mastery is ensured, and by additional later reproductions gain in assurance and ease is secured.

Left to itself every mental content gradually loses its capacity for being revived, or at least suffers loss in this regard under the influence of time. Facts crammed at examination time soon vanish, if they were not sufficiently grounded by other study and later subjected to a sufficient review. But even a thing so early and deeply founded as one's mother tongue is noticeably impaired if not used for several years.

\section{Section 3. Deficiencies in our Knowledge concerning Memory}

The foregoing sketch of our knowledge concerning memory makes no claim to completeness. To it might be added such a series of propositions known to psychology as the following: "He who learns quickly also forgets quickly," "Relatively long series of ideas are retained better than relatively short ones," Old people forget most quickly the things they learned last," and the like. Psychology is wont to make the picture rich with anecdote and illustration. But - and this is the main point even if we particularise our knowledge by a most extended use of illustrative material, everything that we can say retains the indefinite, general, and comparative character of the propositions quoted above. Our information comes almost exclusively from the observation of extreme and especially striking cases. We are able to describe these quite correctly in a general way and in vague expressions of more or less. We suppose, again quite correctly, that the same influences exert themselves, although in a less degree, in the case of the inconspicuous, but a thousand-fold more frequent, daily activities of memory. But if our curiosity carries us further and we crave more specific and dependencies, both those already mentioned and others, if we put questions, so to speak, concerning their inner structure - our answer is silence. How does the disappearance of the ability to reproduce, forgetfulness, depend upon the length of time during which no repetitions have taken place? What proportion does the increase in certainty of reproduction bear to the number of repetitions? How do these relations vary with the greater or less intensity of the interest in the thing to be reproduced? These and similar questions no one can answer.

This inability does not arise from a chance neglect of investigation of these relations. We cannot say that tomorrow, or whenever we wish to take time, we can investigate these problems. On the contrary this inability is inherent in the nature of the questions themselves. Although the conceptions in question - namely, degrees of forgetfulness, of certainty and interest - are quite correct, we have no means for establishing such degrees in our experience except at the extremes, and even then we cannot accurately limit those extremes. We feel therefore that we are not at all in a condition to undertake the investigation. We form certain conceptions during striking experiences, but we cannot find any realisation of them in the similar but less striking experiences of everyday life. Vice versa there are probably many conceptions which we have not as yet formed which would be serviceable and indispensable for a clear understanding of the facts, and their theoretical mastery.

The amount of detailed information which an individual has at his command and his theoretical elaborations of the same are mutually dependent; they grow in and through each other. It is because of the indefinite and little specialised character of our knowledge that the theories concerning the processes of memory, reproduction, and association have been up to the present time of so little value for a proper comprehension of those processes. For example, to express our ideas concerning their physical basis we use different metaphors-stored up ideas, engraved images, well-beaten paths. There is only one thing certain about these figures of speech and that is that they are not suitable.

Of course the existence of all these deficiencies has its perfectly sufficient basis in the extraordinary difficulty and complexity of the matter. It remains to be proved whether, in spite of the clearest insight into the inadequacy of our knowledge, we shall ever make any actual progress. Perhaps we shall always have to be resigned to this. But a somewhat greater accessibility than has so far been realised in this field cannot be denied to it, as I hope to prove presently. If by any chance a way to a deeper penetration into this matter should present itself, surely, considering the significance of memory for all mental phenomena, it should be our wish to enter that path at once. For at the very worst we should prefer to see resignation arise from the failure of earnest investigations rather than from persistent, helpless astonishment in the face of their difficulties. 